\title{
Evaluation of Sandwich ELISA Galactomannan Test in Samples of Positive LA Test and Positive Aspergillus Antibody
}

\author{
Shigefumi Maesaki, Sumio Kawamura, Kohji Hashiguchi, Mohammad Ashraf Hossain, Eisuke Sasaki, \\ Yoshitsugu MiYazaki, Kazunori Tomono, Takayoshi Tashiro and Shigeru KoHNo
}

\begin{abstract}
Objective The detection of circulating Aspergillus galactomannan antigen is a useful tool for serodiagnosis of aspergillosis. However, the latex agglutination test for the detection of galactomannan is not completely reliable due to it's low sensitivity. The sandwich ELISA was developed to achieve high sensitivity. Materials The sandwich immunocapture ELISA was evaluated by testing 56 sero-positive and 56 sero-negative samples of circulating galactomannan detected by LA test retrospectively. Results Sixty of the samples were positive for galactomannan as measured by sandwich ELISA. Fifteen samples out of 56 samples negative by LA test were positive by ELISA and 4 samples out of 56 samples positive by LA test were negative by ELISA. Among 47 serum samples positive for anti-Aspergillus antibody, 14 samples were positive by ELISA. Conclusion In conclusion, galactomannan may be detected in more samples of by the new sandwich ELISA than by LA test.
\end{abstract}

(Internal Medicine 38: 948-950, 1999)

Key words: aspergillosis, serodiagnosis, aspergilloma, anti-Aspergillus antibody, invasive aspergillosis

\section{Introduction}

Aspergillus species are ubiquitous fungi and have been implicated as the causative agents of a variety of lung disorders in humans. These disorders include allergic, saprophytic, and systemic manifestations. The allergic disorders mainly occur in atopic persons, and invasive or systemic diseases occur in immunosuppressed individuals (1). Aspergillosis is a major cause of morbidity and mortality in granulocytopenic patients. With the increasing use of aggressive chemotherapy causing prolonged granulocytopenia in patients with cancer, the risk of disseminated fungal infection is increased. Although Aspergillus species are known to be the most common fungal pathogens responsible for disseminated infection, diagnosis of such infection may be difficult (2).

During the past decade, substantial progress has been made in the development of new approaches and methods for the serological diagnosis of the mycoses. Clinically relevant antigens have been adapted for use in immunoassays for the detection of specific antibodies (3), and methods that detect fungal antibody in body fluids have been progressively refined (4). With a growing number of reports on the incidence of aspergillosis and an increase in the number of immunosuppressed individuals in the population, more rapid methods and more reliable reagents for immunodiagnosis are needed.

Serodiagnosis relies on the detection of circulating antigens produced by Aspergillus fumigatus and other Aspergillus species. Galactomannan (GM) is one such antigen that is measurable in sera from patients with aspergillosis (5). Most assays to detect antigenemia use latex agglutination but its sensitivity does not appear to be sufficient (6). To improve the sensitivity of GM detection, a double direct sandwich ELISA was designed with the same monoclonal antibody as acceptor and detector, which recognizes the galactofuran side chain of the GM molecule (7).

In this study, we evaluated the usefulness of an enzymelinked immunosorbant assay (ELISA) for the detection of galactomannan antigen and compared the results with those of latex agglutination. We also assessed the usefulness of the sandwich ELISA test for the detection of circulating galactomannan in anti-Aspergillus antibody-positive serum samples.

\section{Materials and Methods}

\section{Serum samples}

A total of 112 serum samples were obtained from patients

From the Second Department of Internal Medicine, Nagasaki University School of Medicine, Nagasaki

Received for publication December 3, 1998; Accepted for publication August 5, 1999

Reprint requests should be addressed to Dr. Shigeru Kohno, the Second Department of Internal Medicine, Nagasaki University School of Medicine, Sakamoto 1-7-1, Nagasaki 852-8501 
in Nagasaki University Hospital from 1992 to 1997. Seventythree samples were from patients with aspergillosis, pulmonary aspergilloma (54 samples), invasive pulmonary aspergillosis (8 samples), chronic necrotizing pulmonary aspergillosis (3 samples), aspergillus pyothorax (3 samples), allergic bronchopulmonary aspergillosis (3 samples), and aspergillus fungemia ( 2 samples). The diagnosis of pulmonary aspergilloma was made based on the presence of a fungus ball detected on the chest X-ray, a positive result for aspergillus antibody in the serum, and isolation of Aspergillus spp. from the sputum or bronchial aspirate. The diagnosis of invasive pulmonary aspergillosis was diagnosed by the histopathological findings for the lung or isolation of Aspergillus spp. from the sputum or by the sudden appearance of infiltration shadows in immunosuppressed patients, despite treatment with broad-spectrum antibiotics. The diagnosis of aspergillus pyothorax was made based on the isolation of Aspergillus spp. from a sample of pleural effusion. The diagnosis of allergic bronchopulmonary aspergillosis was done according to the criteria reported by Rosenberg et al (8). Chronic necrotizing pulmonary aspergillosis was diagnosed by histopathological findings for the lung in the patients with mild immunosuppression or underlying lung diseases. The diagnosis of aspergillus fungemia was based on the isolation of Aspergillus spp. from the blood culture.

Thirty-nine serum samples were from the patients with diagnoses other than aspergillosis, consisting of lung cancer (6 samples), pulmonary fibrosis (5 samples), bacterial pneumonia (5 samples), atypical mycobacteriosis (2 samples), pulmonary cryptococcosis (6 samples) and candidemia (2 samples), and patents with hemosputum (5 samples) and healthy volunteers ( 8 samples). Sera were kept at $4^{\circ} \mathrm{C}$ for testing (within 48 hours), then stored at $-20^{\circ} \mathrm{C}$. The sandwich immunocapture ELISA was evaluated by testing 56 sero-positive and 56 seronegative samples of circulating galactomannan, as detected by LA test, and 47 antibody-positive serum samples from the 44 patients with pulmonary aspergilloma and 3 patients with allergic bronchopulmonary aspergillosis.

\section{Detection of Aspergillus galactomannan antigen}

Circulating Aspergillus galactomannan was detected using both latex agglutination (LA) test (Pastorex Aspergillus, Sanofi Diagnostics Pasteur, France) and a sandwich immunocapture ELISA (Platelia Aspergillus, Sanofi Diagnostics Pasteur). For both techniques, immune-complexes were disrupted and proteins were precipitated by adding $100 \mu \mathrm{l}$ of treatment solution to $300 \mu \mathrm{l}$ of undiluted serum in a tightly closed Eppendorf tube which was heated to $100^{\circ} \mathrm{C}$ for 3 minutes, then centrifuged at $10,000 \mathrm{~g}$ for 10 minutes. The supernatant was used for both tests.

For the LA test, $40 \mu \mathrm{l}$ of the supernatant of each was transferred onto disposable plastic cards. Ten microliters of the latex reagent was added and mixed with the supernatant using mixing sticks. The results were recorded after 10 minutes of rotation at $120 \mathrm{rpm}$ on a minirotor. A galactomannan control of $75 \mathrm{ng} / \mathrm{ml}$ was included with the kit and tested with each batch of serum. Whenever positive results were obtained, two-fold dilutions were used.

For the ELISA technique, all reagents were provided by the manufacturor. The sensitized microtiter plates were filled with $50 \mu \mathrm{l}$ of horseradish peroxidase-conjugated monoclonal antibody-galactomannan MAB Eb-A2 as the capture and detector antibody, followed by $50 \mu \mathrm{l}$ of the pretreated serum supernatant. Plates were incubated for 90 minutes at $37^{\circ} \mathrm{C}$. After thorough washings for 5 times, the reaction was allowed to incubate for 30 minutes in darkness with $200 \mu$ l of buffer containing $o$-phenylenediamine. The optical density (OD) was read at $450 / 620 \mathrm{~nm}$. The threshold of the positive sample was estimated to be approximately $1 \mathrm{ng} / \mathrm{ml}$ of galactomannan in human serum. The results were calculated by the following ratio for each test serum: I (cut off index)=OD sample/OD threshold serum. The results were estimated as follows; $I \geqq 1.5$ positive, $1 \leqq \mathrm{I}<1.5$ gray zone, $\mathrm{I}<1$ negative.

\section{Detection of anti-Aspergillus antibody}

The detection of anti-Aspergillus antibody was performed by the double-gel diffusion assay for the precipitating antibodies to Aspergillus fumigatus (Aspergillus Immunodiffusion System, Mercia Diagnosis, Surrey, UK). The test was performed according to the procedure recommended by the manufacturor. The semi-quantitative titer of antigen was determined by the presence of lines of precipitin appearing between the wells corresponding to the $20 \mathrm{mg} / \mathrm{ml} \mathrm{(+)} \mathrm{and} 2 \mathrm{mg} / \mathrm{ml}(2+)$ antigen levels.

\section{Results}

A total of 112 samples were indicated in this study for the detection of galactomannan antigen to compare the usefulness of latex agglutination test and the ELISA assay. Fifteen samples (26.8\%) were positive by ELISA in the 56 negative samples by LA test. Fifteen samples from the patients with pulmonary aspergilloma (11 samples), chronic necrotizing pulmonary aspergillosis ( 2 samples) and aspergillus pyothrax ( 2 samples) were negative by the LA test, but positive by ELISA. Four samples $(7.1 \%)$ from 3 patients with pulmonary aspergilloma and 1 patient with pulmonary fibrosis were negative by ELISA among 56 positive by LA test. Four samples were estimated to be negative by ELISA in 20 samples showing titers of 1 and 2 in the latex agglutination test. There was no negative sample by ELISA in the sera with the titer $>1: 4$ of LA test (Table 1).

The number of the positive galactomannan antigen in the patients with various different forms of aspergillosis is summarized in Table 2 . Among 47 anti-Aspergillus antibody positive sera, 14 samples (29.8\%) (11 from pulmonary aspergilloma and 3 from chronic necrotizing pulmonary aspergillosis) were positive by ELISA. Nine samples $(26.5 \%)$ were evaluated as positive in 34 antibody-positive titer as + , and 5 samples $(38.5 \%)$ were positive in 13 antibody-positive titer as $2+$ (Table 2). 
Table 1. Detection of Circulating Galactomannan Antigen Using Latex Agglutination and Sandwich ELISA Test

\begin{tabular}{lcccc}
\hline \multirow{2}{*}{$\begin{array}{l}\text { Titer of latex } \\
\text { agglutination }\end{array}$} & \multicolumn{3}{c}{ Sandwich ELISA $^{\mathrm{a}}$} & Total \\
\cline { 2 - 4 } & Positive & Gray zone & Negative \\
\hline 0 & 15 & 13 & 28 & 56 \\
1 & 12 & 6 & 2 & 20 \\
2 & 17 & 1 & 2 & 20 \\
4 & 8 & 0 & 0 & 8 \\
8 & 5 & 0 & 0 & 5 \\
16 & 3 & 0 & 0 & 3 \\
\hline Total & 60 & 20 & 32 & 112 \\
\hline
\end{tabular}

${ }^{a}$ The results were calculated from the following ratio for each test serum: I=OD sample/OD threshold serum. The results were estimated as follows; $\mathrm{I} \geqq 1.5$ positive, $1 \leqq \mathrm{I}<1.5$ gray zone, $\mathrm{I}<1$ negative.

Table 2. Detection of Circulating Galactomannan Antigen Using Sandwich ELISA Test in Antibody-positive Samples

\begin{tabular}{ccccc}
\hline \multirow{2}{*}{$\begin{array}{c}\text { Aspergillus } \\
\text { antibody }\end{array}$} & \multicolumn{3}{c}{ Sandwich ELISA } & Total \\
\cline { 2 - 4 } & Positive & Gray zone & Negative & \\
\hline+ & 9 & 13 & 12 & 34 \\
$2+$ & 5 & 1 & 7 & 13 \\
\hline Total & 14 & 14 & 19 & 47 \\
\hline
\end{tabular}

${ }^{a}$ The semi-quantitative titer of antigen was determined by the presence of a series precipitin lines appearing at the $20 \mathrm{mg} / \mathrm{ml}(+)$ and 2 $\mathrm{mg} / \mathrm{ml}(2+)$ antigen levels.

\section{Discussion}

It is difficult to make an early diagnosis of invasive pulmonary aspergillosis, because the sensitivity of the latex assay for detection of galactomannan antigen does not appear to be sufficient (9). The latex agglutination test yields positive results only during the advanced stages of infection in most patients with suspected invasive pulmonary aspergillosis, and dose not contribute to an early diagnosis. A new sensitive double-sandwich enzyme-linked immunosorbent assay can detect less than $1 \mathrm{ng}$ of galactomannan per $\mathrm{ml}$, and can be useful in presumptive diagnosis for the early initiation of antifungal therapy and monitoring treatment in clinically documented invasive pulmonary aspergillosis (10). However, in the present study, four samples of sera which were positive by latex agglutination were negative by ELISA. This discrepancy could be due to falsepositive results by the latex agglutination test, because the results of the latex agglutination test were evaluated subjectively. It was also suggested that there was something inhibiting the reaction of ELISA in these serums. Further studies are needed to clarify this problem.

Pulmonary aspergilloma is typically diagnosed by chest radiography, which shows a fungus ball in the cavity of patients with old pulmonary tuberculosis. More than $80 \%$ of the patients show a positive antibody of Aspergillus fumigatus as tested by immunodiffusion method. However, mycetoma usually harbors the colonized filamentous fungi, which do not invade the lung tissue. The positive samples tested by ELISA in the antibody-positive sera indicated that some patients with pulmonary aspergilloma shown transient galactomannan antigenemia. This result suggested that Aspergillus spp. was not only colonized but possibly invaded the lung tissue. The prognosis of the patients with positive galactomannan tended to be poor based on the results of a retrospective clinical study of the patients with pulmonary aspergilloma (data not shown). A prospective study of the detection of Aspergillus galactomannan by ELISA is now under way, and the usefulness of this means of detection for the prediction of the prognosis of patients with pulmonary aspergilloma may be revealed in a future study.

\section{References}

1) Andriole VT. Aspergillus infections: problems in diagnosis and treatment. Infect Agents Dis 5: 47-54, 1996 (see comments).

2) Bennett JE. Rapid diagnosis of candidiasis and aspergillosis. Rev Infect Dis 9: 398-402, 1987.

3) de Repentigny L. Serological techniques for diagnosis of fungal infection. Eur J Clin Microbiol Infect Dis 8: 362-375, 1989.

4) Hearn VM, Pinel C, Blachier S, Ambroise-Thomas P, Grillot R. Specific antibody detection in invasive aspergillosis by analytical isoelectrofocusing and immunoblotting methods. J Clin Microbiol 33: 982-986, 1995.

5) Latge JP, Kobayashi H, Debeaupuis JP, et al. Chemical and immunological characterization of the extracellular galactomannan of Aspergillus fumigatus. Infect Immun 62: 5424-5433, 1994.

6) Rohrlich P, Sarfati J, Mariani P, et al. Prospective sandwich enzyme-linked immunosorbent assay for serum galactomannan: early predictive value and clinical use in invasive aspergillosis. Pediatr Infect Dis J 15: 232 237, 1996.

7) Verweij PE, Rijs AJ, De Pauw BE, Horrevorts AM, Hoogkamp-Korstanje JA, Meis JF. Clinical evaluation and reproducibility of the Pastorex Aspergillus antigen latex agglutination test for diagnosing invasive aspergillosis. J Clin Pathol 48: 474-476, 1995.

8) Rosenberg M, Patterson R, Roberts M. Immunologic responses to therapy in allergic bronchopulmonary aspergillosis: serum IgE value as an indicator and predictor of disease activity. J Pediatr 91: 914-917, 1977.

9) Hopwood V, Johnson EM, Cornish JM, Foot AB, Evans EG, Warnock DW. Use of the Pastorex aspergillus antigen latex agglutination test for the diagnosis of invasive aspergillosis. J Clin Pathol 48: 210-213, 1995.

10) Sulahian A, Tabouret M, Ribaud P, et al. Comparison of an enzyme immunoassay and latex agglutination test for detection of galactomannan in the diagnosis of invasive aspergillosis. Eur J Clin Microbiol Infect Dis 15: 139-145, 1996. 